\title{
Attitude and willingness of attendance for participating in or completing acupuncture trials: a cross-sectional study
}

This article was published in the following Dove Press journal:

Patient Preference and Adherence

\author{
Xin-lin $\mathrm{Li}^{1}{ }^{1, *}$ \\ Hui-juan Caol,* \\ Ya-jing Zhang' \\ Rui-xue Hu' \\ Bao-yong Lai' \\ Nan-qi Zhao' \\ $\mathrm{Hui} \mathrm{Hu}{ }^{2}$ \\ Zhan-guo $\mathrm{Xie}^{3}$ \\ Jian-Ping Liu'
}

'Centre for Evidence-Based Chinese

Medicine, Beijing University of

Chinese Medicine, Chaoyang

District, Beijing I00029, China; ${ }^{2}$ The

Department of Acupuncture and

Metabolic Diseases, Eastern Hospita

Affiliated to Beijing University of

Chinese Medicine, Fengtai District,

Beijing, China; ${ }^{3}$ The Department of

Acupuncture and Moxibustion, First

People's Hospital of Dongcheng

District, Beijing, China

*These authors contributed equally to this work
Correspondence: Jian-Ping Liu

Center for Evidence-Based Chinese

Medicine, Beijing University of Chinese

Medicine, I I North 3rd East Road,

Chaoyang District, Beijing 100029, China

Email jianping_@@hotmail.com
Objective: To explore the influence of patients' participation in and completing the acupuncture clinical trials through a cross-sectional survey. In addition, we explored potential factors involved in improving patient's compliance to treatment, thus enhancing the quality of acupuncture clinical studies.

Methods: A survey was conducted at outpatient department of acupuncture and metabolic diseases in two hospitals in Beijing. The semi-structured questionnaire was designed based on literature review and Delphi methods. It contains 15 questions related to patients' experience and attitude. SPSS 22.0 was used for analyses. OR and 95\% CI were used for dichotomous outcomes. Logistic regression analysis (LRA) and multi-LRA were used to explore the factors influencing patients' participation or completion and the relationship between demographic characteristics and potential factors.

Results: A survey was conducted from April to September 2016. Five hundred patients were consecutively sampled to fill semi-structured questionnaires regardless of their types of disease. The participants (75.2\% were female) were in the age range of 15-85 years and all of them completed the survey. The effect and safety of acupuncture therapy were considered to be the deciding factors by $92 \%$ and $96 \%$ of the respondents, respectively. Only 40 of the surveyed participants $(8.0 \%)$ had previously participated in the clinical trials. The LRA showed they paid more attention to treatment regimen (frequency and session of treatment) when deciding whether or not to participate in the trials (OR 1.54, 95\% CI 1.02-2.34). Multivariate LRA showed that elder people considered cost (OR 1.36, 95\% CI 1.09-1.70) to be an important factor, while the participants having medical insurance (OR 1.45, 95\% CI -0.20-0.93) thought informed consent was important. Meanwhile, participants with higher education preferred regular follow-up (OR 1.16, 95\% CI 0.02-0.28).

Conclusion: After providing adequate information regarding the potential benefits and harms of the acupuncture treatment, completion of the treatment within the specific time regimen was found to be the most important factor affecting patient's compliance. Other factors, such as cost and regular follow-up, should also be given special consideration.

Keywords: patient compliance, acupuncture, clinical trials, cross-sectional study

\section{Introduction}

Patients' compliance toward the clinical study mainly refers to whether the patients complete the studies according to the protocol. ${ }^{1}$ One of the important indexes used to evaluate the quality of clinical randomized controlled trials and the prognosis of disease is the completion of the study data, which in turn is related to the compliance 
of patients. ${ }^{2}$ Thus, the improvement of the quality of clinical study also requires coordination from the subjects.

The inconsistency between patients' preference and the randomized treatment might affect patient compliance. ${ }^{3}$ We searched one electronic database in China to estimate the number of published clinical trials on acupuncture and found that only $33.3 \%$ of the patients completed the study. ${ }^{4}$ This might be related to the poor level of scientific and health education concerning this approach in the general population. ${ }^{5}$ Besides, this may also be related to the patients' attitude toward acupuncture clinical trials. For example, patients may change their decision to participate in the trial due to many reasons, such as based on the information they might have received ${ }^{6,7}$ regarding the safety of interventions, the time constraints, or personal interests. ${ }^{8-13}$ One study reported two-thirds of older Chinese adults had difficulty in reading and understanding, ${ }^{14}$ which might have also affected the choice and their performance when involved in a clinical trial.

From the literature search, we came across 343 acupuncture trials which reported on the causes for dropout. We conducted a review ${ }^{15}$ to explore the reasons for dropout based on the information provided in those trials. It was found that loss of patients in an acupuncture trial were mainly due to the reason that participants used other interventions during the clinical trial without authorization, occurrence of adverse events, or they were not satisfied with the treatment arrangement or efficacy. In addition, business trip, house moving, or economic reasons may also lead to dropout. On the other hand, a small number of participants may choose to terminate the treatment if they felt better than before.

With acupuncture becoming more and more popular, rigorously designed trials are needed to provide valid evidence for the effectiveness and safety of acupuncture treatment. However, recent studies are more focused on the methodological quality to ensure that an appropriate protocol is followed. Unfortunately, the reasons as to why patients quit non-drug (eg, acupuncture and cupping) clinical trials remain unclear. ${ }^{16}$ If the patients could not comply with the protocol treatment, they may be influenced by unclear factors during the treatment.

In previous studies, the methodology adopted in acupuncture clinical trials mainly placed emphasis on the design of the study or training provided for the researchers. ${ }^{17,18}$ The studies that explored the factors affecting the patient's participation in and/or completing the trials are rare. In order to increase the patients' compliance and further improve the quality of studies, we explored the factors affecting the patients' participation in acupuncture clinical trial from their perspective through cross-sectional survey.

\section{Methods \\ Study design}

A survey was conducted at outpatient department of acupuncture and metabolic diseases in two integrative medicine hospitals in Beijing. The semi-structured questionnaire was designed based on literature review and Delphi methods. It contains 15 questions related to patients' experience and attitude toward participating in acupuncture trials. The aim of this study was to explore the influence of factors that affect patients' participation in and completing the acupuncture trials through a cross-sectional survey. Additionally, we explored the potential methods for improving patient's compliance.

\section{Study subjects}

A survey was conducted at the Department of Acupuncture and Metabolic Diseases in Dongfang Hospital of Beijing University of Chinese Medicine and the Department of Acupuncture and Moxibustion in the First People's Hospital of Dongcheng District during the period from April to July in 2016. The survey enrolled patients over 14 years of age who visited the above-mentioned departments. Patients were consecutively recruited to fill the self-administered semi-structured questionnaires regardless of their types of disease. The survey aimed to improve the patients' compliance to acupuncture clinical trials which may in turn help to increase the quality of acupuncture trials by understanding the important factors from patient's point of view. All participants provided written informed consent, and the participants under the age of 18 years had written informed consent from their parent or legal guardian.

\section{Questionnaires and sample size}

Through the literature review, we summarized several themes related to acupuncture clinical trials, such as experience of acupuncture, cognition of clinical trials, and the experience of participating in clinical trials. Based on this information, we designed the questionnaire comprising 15 questions and sent them to seven experts in the field of acupuncture and methodology. Likert scale was used to assess their attitudes about the design of the questionnaire. Then, we revised the questionnaire according to the experts' feedback. A modified questionnaire was delivered to the experts to score it again. 
Items those had point of disagreement from more than three experts were modified or even deleted.

We investigated 20 patients and made minor modifications to the language of the survey questions according to the pre-survey. The final version of the questionnaire was semi-constructed with 15 questions related to the patients' knowledge of clinical trials. Components of the survey included five parts: general information (demographic characteristics data of the participants: age, sex, nationality, education level, and income); experience with acupuncture treatment; the cognition of clinical trials; the experience of participation in clinical trials; and potential factors influencing their compliance (Figure S1).

The sample size was calculated according to the number of questions. By 30 times the number of the questions, and considering additional $10 \%$ possible dropout rate during the study, 500 cases were expected to be investigated.

\section{Collecting data}

The questionnaires were planned to be completed in hospitals. After obtaining informed consent, each participant was allocated a unique ID number and was asked to fill the questionnaire themselves. Investigators assisted those patients who could not finish the questionnaire autonomously. All three investigators who had knowledge about the evidencebased medicine and clinical epidemiology courses were trained before the study.

\section{Bias control}

In order to reduce the potential selection bias from a nonrandom sampling survey, we collected the data consecutively. Training for the surveyors and a pilot survey (with 20 participants) was conducted to avoid information bias.

\section{Data analysis}

Epidata software version 3.1 was used to record and analyze the data. Four authors (ZYJ, HRX, LBY, and ZNQ) handled the data analysis. Two of them input the data independently, and another two were to ensure the accuracy. One of them performed the statistical analysis, while others made sure the results were complete and accurate. All of them were blinded and did not know the treatment allocation pattern, the aim of the study, and the enrollment procedure of respondents.

The data were imported into SPSS software (version 22.0) by double entry. The descriptive statistics were conducted based on the demographic characteristics of participants, and then grouped by the potential factors influencing patients' participation in acupuncture trials. Frequency, percentage, median, range, mean, $\mathrm{SD}$, and chi-squared test were applied for the description of variables. OR and its CI were used for dichotomous outcomes. Logistic regression analysis (LRA) was used to explore factors influencing patients' decision, and multivariate LRA was used to explore relationship between demographic characteristics and potential influence factors affecting patient's decision.

\section{Ethics statement}

The design of the study strictly complied with the ethical requirements of the Declaration of Helsinki. The study was approved by the ethics committee of Beijing University of Chinese Medicine (approval number: 2017BZHYLL0316).

\section{Results}

\section{Clinical trial portfolio and patients' recruitment}

Between April and July 2016, 500 patients received semistructured questionnaires. All of them completed the survey without any dropouts. Age of the respondents ranged from 15 to 85 years with an average of 45.85 years $(S D=15.43)$. The youngest participant was only 15 years old, and there were only seven patients $(1.4 \%)$ in the age range of 15-22 years, indicating that majority of the respondents were not students. The respondents included 376 females (75.2\%) and 124 males $(24.8 \%)$. The characteristics of patients are shown in Table 1.

We enrolled patients regardless of the type of disease/ condition and their medical history. There were 268 patients having the history of acupuncture treatment, and only 40 participants $(8.0 \%)$ had previous experience of involvement in clinical trials.

\section{The influence of acupuncture treatment experience on clinical trial participation}

A total of 268 respondents (53.6\%) had received acupuncture treatment before. The major diseases for which they took treatment were cervical spondylosis, back pain, stomach pain, cough, etc. Compared to the patients who had never accepted acupuncture treatment before, those who had acupuncture treatment experience had no significant statistical difference on clinical trial participation experience $(P=0.24>0.05)$. However, patients who had received acupuncture treatment before were more willing to participate in clinical trial of acupuncture initially (OR 0.48 , 95\% CI 1.56-1.68).

\section{The patients' knowledge of clinical trial}

A total of 321 patients had prior knowledge about the concept of clinical trial, and we further investigated about their 
Table I Demographic information of 500 participants

\begin{tabular}{|c|c|c|c|c|}
\hline Characteristics & $\begin{array}{l}\text { Participation } \\
\text { experience (n) }\end{array}$ & $\begin{array}{l}\text { Non-participation } \\
\text { experience }(n)\end{array}$ & Total (n, \%) & $P$-value ${ }^{a}$ \\
\hline $\begin{array}{l}\text { Age (years) } \\
\text { Mean (SD) }\end{array}$ & $44.93(16.40)$ & $45.93(15.35)$ & - & 0.65 \\
\hline Nationality & & & & 0.93 \\
\hline Han & 39 & 446 & $485(97.0)$ & \\
\hline Hui & I & 7 & $8(1.6)$ & \\
\hline Manchu & 0 & 5 & $5(1.0)$ & \\
\hline Mongolian & 0 & 1 & $\mathrm{I}(0.2)$ & \\
\hline Yao & 0 & I & $\mathrm{I}(0.2)$ & \\
\hline Gender & & & & 0.46 \\
\hline Female & 32 & 344 & $376(75.2)$ & \\
\hline Male & 8 & 116 & $124(24.8)$ & \\
\hline Education & & & & 0.74 \\
\hline Junior high education & 4 & 68 & $72(14.4)$ & \\
\hline High school education & 8 & 113 & $|2|(24.2)$ & \\
\hline Junior college degree & 8 & 66 & $74(14.8)$ & \\
\hline Undergraduate & 14 & 160 & $174(34.8)$ & \\
\hline Postgraduate & 6 & 53 & $59(11.8)$ & \\
\hline Income (yuan) & & & & 0.42 \\
\hline$\leq 2,000$ & 7 & 44 & $51(10.2)$ & \\
\hline $2,000-4,000$ & 16 & 182 & $198(39.6)$ & \\
\hline $4,000-5,000$ & 4 & 61 & $65(13.0)$ & \\
\hline$\geq 5,000$ & 13 & 173 & $186(37.2)$ & \\
\hline Medical insurance & & & & 0.33 \\
\hline Medical insurance & 30 & 386 & $416(83.2)$ & \\
\hline Public medical treatment & 3 & 19 & $22(4.4)$ & \\
\hline Self-expense & 7 & 55 & $62(12.4)$ & \\
\hline
\end{tabular}

Note: ${ }^{P}$-values compare the proportion of patients who had participated in clinical trials with patients who had no participation experience.

opinion on the potential benefit/harm associated with participation in a clinical trial. The results are shown in Table 2.

Another 179 patients had no idea of the concept of clinical trial, but most of them had their own understandings. Thirty-five of them (18.4\%) thought clinical trial was aimed to develop new drugs, and 64 of them (35.8\%) thought that a trial may help in the testing of a new therapy for a specific disease. In contrast, other 35 participants (18.4\%) thought patients would be treated as experimental mice in the clinical trials, and that researchers cannot guarantee the safety of the treatment. Twelve respondents were of opinion that the clinical study was required for scientific research, it could

Table 2 Opinion of 321 patients on the benefit/harm from participating in clinical trials

\begin{tabular}{l|l}
\hline Potential benefits/harm & $\begin{array}{l}\text { Number of the } \\
\text { patients }(\%, \mathbf{n = 3 2} \text { I) }\end{array}$ \\
\hline Better effect of treatment & $108(33.6)$ \\
Free treatment & $99(30.8)$ \\
Uncertain about good effect & $9 \mathrm{I}(28.3)$ \\
Comprehensive and meticulous & $16(5.0)$ \\
diagnosis and treatment & $7(2.3)$ \\
No effect, maybe has side effect &
\end{tabular}

be used to evaluate the effect of a treatment, or it was just to popularize the target treatment. The remaining 37 patients did not comment on it.

\section{The potential influential factors affecting patients' participation in acupuncture clinical trial}

Participants were asked to evaluate the importance of the following factors when considering to join an acupuncture clinical trial: treatment expenses, treatment regimen (frequency and session of treatment), the degree of understanding of the informed consent, effectiveness, safety, and whether the follow-up is timely. The importance was also classified as unimportant, indifferent, more important, and very important, and Table 3 describes the constituent ratio of respondents' attitude toward each item. Results of the investigation showed that patients' attitude toward expenses were flat on four degrees of cognition. However, for the other five aspects, such as treatment regimen, fully informed consent, effect of treatment, safety during the acupuncture clinical trials, and follow-up in time, the degree of attention was increasing separately (Table 3). 
Table 3 Factors influencing patients' participation in clinical trials

\begin{tabular}{l|l|l|l|l}
\hline Factors & $\begin{array}{l}\text { Not important } \\
(\mathbf{n}, \%)\end{array}$ & $\begin{array}{l}\text { Indifferent } \\
(\mathbf{n}, \%)\end{array}$ & $\begin{array}{l}\text { Slightly important } \\
(\mathbf{n}, \%)\end{array}$ & $\begin{array}{l}\text { Very important } \\
(\mathbf{n}, \%)\end{array}$ \\
\hline Cost of the treatment & $94(18.8)$ & $190(38.0)$ & $155(3 I .0)$ & $61(12.2)$ \\
Treatment regimen & $32(6.4)$ & $137(27.4)$ & $205(4 I .0)$ & $126(25.2)$ \\
Fully informed consent & $17(3.4)$ & $37(7.4)$ & $225(45.0)$ & $22 I(44.2)$ \\
Effect of treatment & $5(1.0)$ & $37(7.4)$ & $195(39.0)$ & $263(52.6)$ \\
Safety during the clinical trials & $\mathrm{I}(0.2)$ & $17(3.4)$ & $120(24.0)$ & $362(72.4)$ \\
Follow-up in time & $8(1.6)$ & $126(25.2)$ & $199(39.8)$ & $167(33.4)$ \\
\hline
\end{tabular}

\section{The LRA for factors influencing patients' involvement in acupuncture clinical trials}

In order to explore the different attitude towards the influential factors between respondents those had or not had clinical trial participation experience, we analyzed the data of them by logistic regression analysis (LRA) (Table 4$)$. The result showed there was significant difference $(P=0.04,<0.05)$ between them with regard to "treatment regimen (frequency and session of treatment)". Respondents who had participated in clinical trials earlier paid more attention to "treatment regimen" (OR 1.54, 95\% CI 1.02-2.34), which indicated that high frequency treatment may reduce the possibility of a patient taking part in a clinical trial.

Furthermore, we explored the difference in the factors that influence the attitude between males and females, and between low-, middle-, high-income groups by LRA. The result showed there was no significant difference with respect to the treatment regimen, fully informed consent, effects of treatment, safety, or timely follow-up.

Furthermore, the multivariate LRA was conducted to explore the relationship between demographic characteristics and potential influential factors. The results showed that elder people considered cost (OR 1.36, 95\% CI 1.09-1.70), treatment regimen (OR $0.55,95 \% \mathrm{CI}-0.81$ to -0.38 ), and timely follow-up (OR $0.92,95 \%$ CI -0.53 to -0.07 ) as more important factors; the participants who had medical insurance (OR 1.45, 95\% CI -0.20-0.93) thought that informed consent was more important. Meanwhile, participants with higher education level preferred regular follow-up (OR 1.16, 95\% CI 0.02-0.28), as well as by those who had medical insurance (OR 2.07, 95\% CI 0.19-1.26) or whose medical expenses were paid by public expense (OR 3.89, 95\% CI 0.34-2.37).

\section{Discussion \\ Primary findings}

In this study, we found that the treatment regimen (time and frequency of treatment), the degree of understanding of the test procedure, treatment plan, effectiveness, and safety of the treatment might affect the patients' decision to participate in a clinical trial. Especially, the time period of the treatment may affect the decision as to whether or not to join in the clinical trial and complete the whole treatment regimen.

It can be concluded that effectiveness and safety were the two factors that were considered important (over $90 \%$ importance), followed by informed consent (89\%). In fact, all the three parameters were closely related, since an informed consent must provide full details regarding both possible effects and safety issues, and for a trial, consent may include all the other factors listed in Tables 3 and 4. Thus, informed consent can be considered as the most important factor, as it covers all the other factors.

Table 4 The LRA between factors influencing patients' involvement and participation experience

\begin{tabular}{l|l|l|l|l}
\hline Variable & $\mathbf{B}^{\mathbf{a}}$ & OR & $\mathbf{( 9 5 \% ~ C l )}$ & $\boldsymbol{P}_{\text {-value }}$ \\
\hline Cost of the treatment & -0.19 & 0.83 & $(0.56,1.23)$ & 0.34 \\
Treatment regimen & 0.43 & 1.54 & $(1.02,2.34)$ & 0.04 \\
Fully informed consent & -0.29 & 0.75 & $(0.37,1.50)$ & 0.41 \\
Effectiveness & 0.13 & 1.14 & $(0.64,2.04)$ & 0.65 \\
Safety & -0.05 & 0.95 & $(0.44,2.04)$ & 0.89 \\
Follow-up in time & -0.03 & 0.97 & $(0.59,1.57)$ & 0.89 \\
Constant & 3.03 & 20.69 & - & 0.04 \\
\hline
\end{tabular}

Note: ${ }^{a}$ Represents regression coefficient.

Abbreviation: LRA, logistic regression analysis. 


\section{Strengths and weaknesses of the study}

Previous studies more likely used scales which were graded by the respondents to explore the potential factors affecting patients' compliance. ${ }^{19}$ Some studies focused on the objective factors, such as the impact of family members and medical services. ${ }^{20}$ Most of the clinical trials evaluated the patients' medication compliance by recording the patient diary. ${ }^{21}$ In acupuncture clinical trials, patients' compliance was also reflected in the medical records maintained during the process of treatment. But this would be influenced by researchers' subjective impact on the process of recording, which cannot fully reflect the true intentions of respondents. So this study was aimed to excavate the influential factors based on the subjective views of the interviewees through the cross-sectional survey.

There are some limitations in this study. First, all participants were recruited from the patients visiting the acupuncture departments, which mean they had preference for acupuncture treatment. Thus, the participants may not be the true representatives the general population. Our goal of this study was to explore the factors that influence the patients' participation in and completing the acupuncture clinical trials. To achieve this goal, respondents who have prior experience of participation in a clinical trial should be included, since they will have better knowledge of the whole process of trial and the problems encountered during the process of treatment. But in this study, though the sample size is powerful enough, the number of respondents who had participated in a clinical trial before was relatively small (only $8 \%$, which may not be sufficient to reflect upon the viewpoints of the experienced patients. Because of this, we could only make conclusions on the potential influence of patients' participation in but not completing the acupuncture clinical trial. Furthermore, the preponderance of females (2:1) may also affect the apparent importance of influencing factors. To avoid the above-mentioned potential selection bias, subjects should be randomly selected from general populations for the conductance of this type of study in the future.

Secondarily, one of the investigators assisted the subjects to fill in the questionnaire. Though this was probably necessary for some of the subjects, and the investigators were trained before the survey, there is still a danger that the investigator might have projected his own opinion to encourage the subject. This may lead to potential information bias.

\section{Implications for physicians, policymakers, and future research}

Majority of the patients are concerned about the effectiveness and safety of the treatment, thus fully informed consent before recruitment is quite important and should be mandatory to clarify the potential benefits and harms for those who participate in the trial. Researchers should try their best to explain the items of the consent to the participants, and allow the participants to quit the survey if they request to. This not only prevents unsuitable patients from entering a trial but also avoids their withdrawal before completion, since they will have had all the risks and benefits explained fully before the beginning of the trial. The standard features to be included in the consent for a trial contain, but not limited to, details regarding the type of treatment (possible groups for allocation), frequency and length of treatment, suspected/possible efficacy, risk of side effects/safety, free or cost of treatment/travel expenses, and length of follow-up.

Moreover, free treatment should be provided in a clinical trial to encourage patients, especially patients with no medical insurance, to participate in the program. A regular follow-up during the treatment is needed to record the progress in patient's conditions and attend to the adverse events which may be related to the treatment.

There are still some other factors that influence the compliance of patients, but are not included in the questionnaire, such as the length of the trial, the treatment environment, type of the disease, etc. Future studies may consider exploring these factors.

Since the number of experienced patients in this study is limited, we suggest that an in-depth interview be conducted for respondents who had been involved in clinical trials before to enable exploration of more factors that may have a deeper impact on patients' compliance, which might provide hints to future research and clinical practice.

\section{Conclusion}

After providing adequate information regarding the potential benefits and harms of the acupuncture treatment, completion of the treatment within the specific time regimen was found to be the most important factor affecting patient compliance. Other factors, such as cost of the treatment and regular follow-up, should be given special consideration according to specific demographic characteristics.

\section{Acknowledgments}

The authors extend their thanks to Dr WJ Yang and Dr L Zhou for providing guidance during the survey. This work was partially supported by Capacity Building in Evidencebased Chinese Medicine and Internationalization Project (1000061020008). 


\section{Disclosure}

Hui-juan Cao is supported by the Beijing Municipal Organization Department (2017000020124 G292). The authors report no other conflicts of interest in this work.

\section{References}

1. Liu JP. Analysis on the dependence and intention of experimental randomized controlled therapy. Zhongguo Zhong Xi Yi Jie He Za Zhi. 2003;23(12):884-886

2. Wood AM, White IR, Thompson SG. Are missing outcome data adequately handled? A review of published randomized controlled trials in major medical journals. Clin Trials. 2004;1(4):368-376.

3. Haynes RB, Dantes R. Patient compliance and the conduct and interpretation of therapeutic trials. Control Clin Trials. 1987;8(1):12-19.

4. Zhou JJ, Li YZ. Investigation and countermeasure research of patient compliance. Management of Traditional Chinese Medicine. 2013; 21(11):1170-1172. Chinese.

5. Bostock S, Steptoe A. Association between low functional health literacy and mortality in older: a cross-sectional survey. BMJ. 2012; 344:e1602.

6. Kay A, Higgins J, Day AG, Meyer RM, Booth CM. Randomized controlled trials in the era of molecular oncology: methodology, biomarkers, and end points. Ann Oncol. 2012;23(6):1646-1651.

7. Booth CM, Cescon DW, Wang L, Tannock IF, Krzyzanowska MK. Evolution of the randomized controlled trial in oncology over three decades. J Clin Oncol. 2008;26(33):5458-5464.

8. Joffe S, Cook EF, Cleary PD, Clark JW, Weeks JC. Quality of informed consent in cancer clinical trials: a cross-sectional survey. Lancet. 2001;358(9295):1772-1777.

9. Godskesen T, Hansson MG, Nygren P, Nordin K, Kihlbom U. Hope for a cure and altruism are the main motives behind participation in phase 3 clinical cancer trials. Eur J Cancer Care. 2015;24(1):133-141.

10. Daugherty C, Ratain MJ, Grochowski E, et al. Perceptions of cancer patients and their physicians involved in phase I trials. J Clin Oncol. 1995;13(5):1062-1072.
11. Schroen AT, Petroni GR, Wang H, et al. Preliminary evaluation of factors associated with premature trial closure and feasibility of accrual benchmarks in phase III oncology trials. Clin Trials. 2010;7(4): 312-321.

12. Schroen AT, Petroni GR, Wang H, et al. Achieving sufficient accrual to address the primary endpoint in phase III clinical trials from U.S. Cooperative Oncology Groups. Clin Cancer Res. 2012;18(1):256-262.

13. Stensland KD, Mcbride R, Wisnivesky JP, et al. Premature termination of genitourinary cancer clinical trials. Journal of Clinical Oncology. 2014;32(4 suppl):288.

14. Zhang HK. The status quo and changes of the education level of the elderly population in China. Chinese Journal of Gerontology. 2016;36(5): 1215-1216.

15. Li XL, Cao HJ, Zhang YJ, Hu RX, Liu JP. Current status of missing data in domestic acupuncture clinical trial reports and its influence on research conclusions. Journal of Traditional Chinese Medicine. 2018;59(7):577-581. Chinese.

16. Hollis S, Campbell F. What is meant by intention to treat analysis? Survey of published randomised controlled trials. BMJ. 1999; 319(7211):670-674.

17. Gd L, Zhang LL. Compliance management of the subjects in clinical drug trials. Hosp Adm J Chin PLA. 2008;15(3):215-217.

18. Li Y, Liang FR, Zhao L, Zhang L. The design, implementation, supervision and reporting of multi-centered acupunctural clinical trial. Zhongguo Zhong Xi Yi Jie He Za Zhi. 2009;29(7):652-655.

19. Gf L. Investigation and analysis of the related factors of treatment compliance in patients with schizophrenia. J Nurs Train. 2012;27(10): 926-927.

20. Ln L, Wang Y, Fq L. Investigation of depression and treatment compliance in elderly diabetic patients. J Nurs Train. 2012;27(24): 2233-2235.

21. Shen AZ, Chen FH, Chen LM. Research progress on patients' compliance. Herald Medicine. 2005;24(8):712-714. 


\section{Supplementary material}

\section{Essential information}

Name: Age: Nation: Occupation:

Educational level:
A. Junior high school education
B. High school
C. Undergraduate
D. Junior college
E. Special secondary school
F. Graduate students and above Income (RMB):
A. Less than 2,000
B. $2,000-4,000$
C. $4,000-5,000$
D. More than 5,000

Pay for medical treatment:
A. Medical insurance
B. Public medical treatment
C. Self-expense

\section{The experience of acupuncture}

1. Is it the first time to receive acupuncture and moxibustion?
A. Yes
B. No

2. What diseases is the main treatment?

\section{Cognition of clinical trials}

3. Have you ever heard of clinical trials of acupuncture and moxibustion?
A. Yes
B. No

4. What do you think about acupuncture clinical trial?

5. What do you think is the benefit of participating in an acupuncture trial?
A. Better effect of treatment
B. Free treatment
C. Others (please describe in details)

6. Are you willing to take the initiative to participate in research projects and consult doctors and nurses on clinical issues?
A. Yes
B. No

7. What do you think is key factor for deciding to participate in a clinical trial?
A. Whether the disease is in conformity with the study
B. Treatment arrangement (eg, time)
C. Doctors expertise
D. Others (please describe in details)

8. How the following factors are important for you when deciding to participate in a trial?

\begin{tabular}{l|l|l|l|l}
\hline & Not important & Indifferent & Slightly important & Very important \\
\hline Cost of the treatment & & & & \\
Treatment regimen & & & & \\
Fully informed consent & & & & \\
Effect of treatment & & & & \\
Safety during the clinical trials & & & & \\
Follow-up in time & & & & \\
\hline
\end{tabular}

\section{The experience of participating clinical trials}

9. Have you ever participated in a clinical trial before?
A. Yes
B. No

10. What do you think about the clinical trials that you have been involved in?

11. Where did you hear about the trial you involved?
A. Posters
B. Leaflets, brochures
C. Introduction from doctors and nurses
D. Blog, WeChat, and Internet promotion
E. Propaganda in community

12. What difficulties have you faced during the process of clinical trials?

13. Have you ever had any experience of not completing a clinical trial?
A. Yes
B. No

14. What is the reason for your discontinuation of the trial? (Multiple choice)
A. Tense time
B. Bad effect
C. An unexpected event (please describe in details)
D. Bad manner of researchers
E. Lack of follow-up
F. Other reasons

15. If you have been involved in a trial, what may make you drop out the trial?

Figure SI A survey about patients' willingness and compliance of participating in or completing acupuncture clinical trial (translation edition). 
Patient Preference and Adherence

Dovepress

\section{Publish your work in this journal}

Patient Preference and Adherence is an international, peer-reviewed, open access journal that focuses on the growing importance of patient preference and adherence throughout the therapeutic continuum. Patient satisfaction, acceptability, quality of life, compliance, persistence and their role in developing new therapeutic modalities and compounds to optimize clinical outcomes for existing disease states are major areas of interest for the journal. This journal has been accepted for indexing on PubMed Central. The manuscript management system is completely online and includes a very quick and fair peer-review system, which is all easy to use. Visit http://www. dovepress.com/testimonials.php to read real quotes from published authors.

\footnotetext{
Submit your manuscript here: http://www.dovepress.com/patient-preference-and-adherence-journal
} 